\title{
Embracing Ingenious People Analytics: Case of PepsiCo
}

\author{
Rekha Bishnoi, Poonam, Arora
}

\begin{abstract}
This study tries to explore various aspects of People analytics and how it impacts the functioning and performance of PepsiCo. Human resource (HR) analytics has attracted significant attention worldwide as HR profession is involved in making highly important decision which rely upon data and help in strategy realization. This paper defines the concept of People analytics based on the literature review and offers an understanding of the role it is currently playing in any organization. The Case of PepsiCo reveals various strategies adopted for implementing People Analytics and how it changed all the real-life business cases and continue to improve its performance via HR analytics practice. In addition to this, HR Analytics at PepsiCo has well identified the most effective way of practicing HR with better performance.
\end{abstract}

Key Words: HR analytics, Organization, People analytics, PepsiCo.

\section{INTRODUCTION}

Business has been changed due to digitization. According to Bersin (2013) by Deloitte, 70\% of fortune 1000 company of 2004 does not exist today due to failing to adopt digitization. According to Harvard Business Review (2013), data-driven solutions lead to improved decisions making about Talent, revenues and profits for a number of Board of Directors, CEOs, and CHROs. Employee feedback is becoming an important aspect of transforming the employee feedback and employees' feedback has gain weightage like customer feedback. (Josh Bersin, 2015).

The economic challenges faced by companies in past few years have given rise to the need of data science. Since data leads information and in turn provide better insights to help decision-making. Including data analytics in HR would be no peculiarity. So, HR Analytics' emerging tools are becoming standard parts of doing business. HR analytics puts a clear picture about the impact of emerging situation of business on the talent picture - and vice versa.

Also many of the CHROs have reached the level of insight on their fingertips that they never had imagined. That's the power of HR analytics which make it indispensable for future generation.

The idea of applying data science to people management is prevalent since the early 1900s. The talent analytics combines workforce data with business data to help companies make better business decisions about People.

Revised Manuscript Received on February 12, 2020.

Rekha Bishnoi, Research Scholar, School of Management, The NorthCap University, Gurugram (Haryana), India.

Dr. Poonam Arora, Assistant Professor, The NorthCap University, Gurugram (Haryana), India.
Today generally HR Manager has questions at frontage like whom to hire, whom to fire and what expedites performance, increases retention and customer's satisfaction. In the similar lines, HR Analytics being a data driven platform aids statistically to answer the people related questions. The power of analytics does not end here and with it has put the opinion experience and gut feeling on the edges. Nothings are better than analytics which is capable of explaining how to sustain employee productivity during a merger or acquisition? Who will be the next employee leaving the company, and why? Since data is the key and leads suggestions so such questions seek answer statistically because these are having big impact on the future business strategies.

\section{OBJECTIVES}

a. Clarify the concept of People Analytics and understanding its importance.

b. To understand the problems before People Analytics

c. To understand strategies implemented under People analytic in PepsiCo.

d. To give recommendation for future research regarding People Analytics.

\section{CONCEPT OF PEOPLE ANALYTICS}

People analytics is a part of business analytics. "The extensive use of data, statistical and quantitative analysis, explanatory and predictive models, and fact-based management to drive decisions and actions is called data analytics." (Davenport \& Harris, 2007, p. 7). Being data pervasive in the organization, it can really help people to take agile, accurate and assertive decision and the science behind data analytics about people of organization is termed as People Analytics.

Including data analytics, People analytics has power to appraise the underlying relationship between HR practices and organizational performance outcomes relating to attrition rate, turnover ratio, customer satisfaction rate and profit. As it applies statistical techniques and experimental approaches which are centered on metrics of efficiency, effectiveness and impact, thus the decisions provided by People Analytics are legitimate and reliable for human capital (Lawler, Levenson \& Boudreau, 2004; Boudreau \& Ramstad, 2006).

According to Deloitte's Global Human Capital Trends 2016, People Analytics has become priority for 77 percentages of executives. About 6 out of 10 organizations are working to implement People Analytics in upcoming 5 years. 
Deloitte's report (2016) claims that percentage of companies practicing workforce analytics to predict performance has grown to $44 \%$ in 2016 as against $29 \%$ in 2015. While the impact of business due to people analytics is being measured in 51\% companies. (Deloitte, 2016). All this tends to show that there is significant increase in companies which are adopting People Analytics to assess business outcomes.

Concisely , People analytics is defined as "an evidencebased approach for making more sophisticated decisions on the human side of the business; it is comprised of an array of tools and technologies, ranging from simple reporting of People metrics all the way up to predictive modeling." (Bassi, 2011).

People analytics may use different types of data collection that are related to Workforce statistics, financial ratios regarding People and productivity, measurement of People's values; extent of People's engagement at workplace, measurement of efficiency and effectiveness of HR processes, measurement of investment in special initiatives and programs (Mayo 2006), and competency measurement.

A Typical People Analytical Model may consist of five steps which help in building PEOPLE Analytic approach for any company.

TrackingData: First of all data tracking includes extracting useful data from a number of resource let it be numerical, textual, audio, video or in the form of images. Raw data is transformed into the useful information, followed by loading and fusing with other sources. Finally data is stored into a data warehouse which is capable of storing big data. It also includes Tracking data does not ends here but it also include keeping eye on live data in the contemporary environment.

Analyzing: It includes using Business intelligence tools such as Power BI, Tableau, query analysis, multidimensional analysis and data mining to analyze big data.

Modeling: After the data is analyzed, there arises a need for formulating a model which can do forecasting, optimization by advanced analytics tools. Various descriptive, predictive and prescriptive statistical methods are used to frame models which later help in decision making.

Decision: Taking a decision on existing or newly developed models makes it easy to take decision for repetitive kind of processes. There is need of inclusive collaboration from every aspect of individuals and their proficiency in using decision making tools.

Action: Action is directed by the decision based on the particular organization. For examples new strategy in recruitment and workforce planning depends upon the analysis and results of previous strategy.

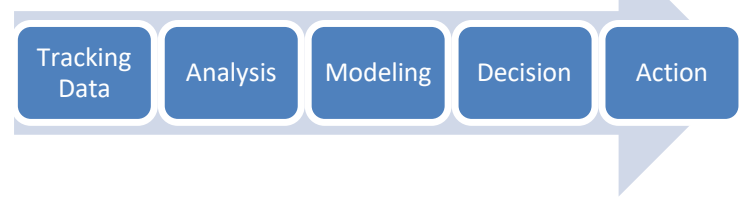

Figure 1
Establishing a relationship between what People does and business outcomes - and then creating strategies based on that information - are what People analytics is all about.

\section{PEOPLE ANALYTICS: AN INSPIRATION TO PEPSICO}

PepsiCo, Inc. is an American food and beverage company and having span across more than 200 countries. When Pepsi-Cola Company merged with Frito-Lay in 1965 it was given a different name i.e. PepsiCo Inc. The company has its headquarters situated in Purchase, New York.

With 263,000 employees PepsiCo is generating over $\$ 63 \mathrm{~B}$ in annual revenues while operating across 200countries. PepsiCo represents an amazing story of transformation and currently at number 45 in the Fortune 500. With People innovations practices, PepsiCo is striving to adopting modern HR practices thus continuously transforming.Likewise, potential practices which will be adopted very soon by PepsiCo are Manager Quality Performance Index (MQPI) and Include, Coach, Appreciate, Respect, and Empathize (ICARE).

Let us understand by an example how PepsiCo harnesses the power of analytics and insights. The company reconciles and forecasts the production and shipment needs as per reports of warehouse inventory and the POS inventory provided by the clients. This way, the company makes certain that retailers have the right products, in the right time and in the right volume by using analytics. In addition to this, with analytics it influences People's decision making about which beverage to pick in the split of second by inspiring them to purchase products with less sugar, sodium, and saturated fat and this is done with just a small change i.e. reorganizing the products in a vending machine. The products are reshuffled in such a manner that they are a more logical fit with People's frame of mind.

\section{ROADBLOCKS FOR IMPLEMENTING PEOPLE ANALYTICS AT PEPSICO}

As per PepsiCo (Total Rewards Conclave, 2017) till 2014 it was using multiple HR systems which did not share information to each other and used by different teams across the world. A simple question about total headcount of PepsiCo all over the world took 8 weeks to answer.

Another problem which Pepsi faced was dealing with different cultures in the international scenario. The level of Diversity and Inclusion (D\&I) was challenge without insufficient data. Adding more to it, Pepsi was also facing external environmental issues like the supply of raw materials to produce their products. Optimum Quantity of raw material and supply was a big challenge in the era of increasing sub brands and retailers. All these challenges led PepsiCo to opt for modern technological practices.

For HR operations team at PepsiCo, it was a trusting moment to deploy cloud HR services across all the countries and today total head count at PepsiCo can be known in only 45 minutes.

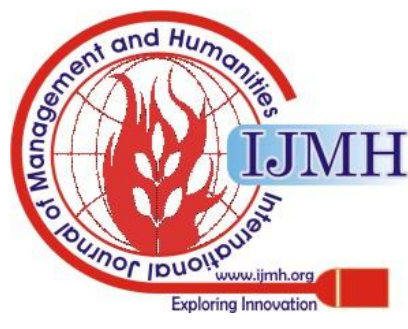




\section{INCEPTION OF PEOPLE ANALYTICS AT PEPSICO}

Integrating each type of the data from various sources and normalizing it seems to be unfeasible sometime when a company like PepsiCo has more than 260,000 employees.

In 2012, the Senior Vice President (SVP) of People operations was asked to digitalize HR. For this, he first managed to bring stakeholders together from across HR, compensation, and IT and faced HR silos around the world which were working on different systems. PepsiCo SVP tried out to integrate the data but faced trouble due to inconsistency in data labels and poor quality of data.

In the way of implementing a robust PEOPLE Analytic framework first thing is identifying the data needed. The second step was to do data taxonomy i.e. data categorization followed by governance rules. This way PepsiCo created a "data lake" named platform-for all PEOPLE data. All this took enormous amount of work including manual data entry into the all-inclusive system. By the end of 2017, people data was accurate and centralized. After that PepsiCo added predictive analytics and AI to enhance its analytical competency, including "robotic process automation". With RPA it saved time and get pay back more than investment.

Today, HR system at PepsiCo is fully digital and has realtime data. (Charan, Barton, and Carey 2018). For this, Pepsico had to invest over $\$ 100 \mathrm{~m}$ into a People transformation initiative focused on harmonizing business processes globally.

\section{SKEPTICISM TO SUCCESS: A ROUTE THROUGH PEOPLE ANALYTICS}

\section{Process Improvement by Robotic Process Automation (RPA)}

RPA is old technology but it really hadn't been used much in People. Robotic Process is benefitting PepsiCo's People processes like recruitment, onboarding and of boarding, data management, compliance, payroll administration, etc. Equally important is the fact that automation frees People working in People departments to focus on the use of their communication skills for proactive hiring, supporting the development of company culture, and other value-added activities.

PepsiCo has used robotic process automation (RPA) in Resume screening and candidate shortlisting, Offer letter administration, Onboarding new hires, Travel and expense management, Employee data management, Attendance tracking, Payroll management, Compliance and reporting. Its advantages thus go beyond financial ones and corroborate the humanist outcome of RPA deployment, the fact that technology makes People really matter.

Interestingly, Robot Vera was the first robot at PepsiCo to make phone calls and interview candidates for open positions. It helped to fill vacancies in sales and as drivers. Also it assisted Russia's HR professionals to fill factory vacancies when they were struggling to fill 250 jobs in just two months. Robot Vera, a product of Russian startup Stafory is competent of interviewing 1,500 candidates in nine hours while humans will take nine weeks for the same.

In addition to this, a user can automatically activate a particular template for the onboarding workflow, and this streamlines the whole process. The bots can then make ruleguided decisions as to which credentials to assign the new employee, which onboarding documents to send etc.

\section{A. A new and better way of onboarding with Appical}

In April 2017, PepsiCo signed a contract to deploy the Appical mobile application to smoothen pre- and onboarding program for the entire procurement department. The Appical application was focused to give plans to support various teams in the marketing, supply chain and others.

PepsiCo University established by PepsiCo overlooks the company's e-learning related to job and training initiatives. As PepsiCo believes, onboarding is a fun and friendly activity but it could be more than just an informative welcome to the company. This application eases preboarding activities, which includes complete information about new hire like names, addresses, social security numbers and emergency contact info. Still onboarding can take up to a month as it includes intensive education on PepsiCo's business operations on a global level.

Appical, which offers the technology and the mobile app to make new employees feel at home quickly and to start with onboarding before starting employment. You are in charge of the content in the application. Using videos, checklists, quizzes, interactive assignments or virtual reality are a common part of an interactive onboarding process for new hires.

Appical provides features such as Dictionary, Checklist, Augmented Reality, Onboard anywhere, Blueprints, People Finder, Interactive Content, Dashboard, Different languages, Manager Dashboard and Chat with colleagues.

Moreover, Appical has cloud-based program which delivers instructional videos, educational webinar, and slideshow-style on "how-to" give presentations with quizzes, workbooks and reference materials. Currently this application supports English, Spanish, Chinese, and Brazilian Portuguese and proposed to include Arabic and Russian language support in the near future.

\section{B. Data Centralization with HR Cloud}

Earlier there were over $70 \mathrm{HR}$ platforms across the globe in PepsiCo except for performance, for learning, for every other process in HR. These entire platform managed data in different way. Due to this inconsistency, it was a big challenge to harmonize data and make it available to the People who needed it.

HR Cloud gives employees the tools to collaborate and grow as well. The employee-centric platform streamlines processes through an intuitive interface and robust technology to help everyone at organization thrive.

\section{ABBYY Flexi Capture-OCR Technology}

Earlier PepsiCo was struck in manual data entry which was creating big inefficiency. Before some time also, its four largest European entities were still reconciling invoice and credit memo data into SAP manually. - The process being a labor-intensive was consuming much time and errorprone also. For this, PepsiCo has opted Imaging Technology that creates image of the document. 
ABBYY Flexi Capture is a business intelligence tool for capturing meaningful data, identifying relationship and generating insights from documents, forms. It also transforms documents into business value by capturing and validating information in any format at its point of need. Intelligently digitizing documents and automating document processes, ABBYY Flexi Capture is a highly accurate and scalable document workflow platform that captures, classifies and transfers critical data from unstructured and structured documents to the right process, workflow or decision engine

\section{Succession Planning}

Pepsico is taking a simple step away with People analytics i.e. shortening the time frame to manage the talent. Instead of usual annual updates, it yields brief updates on the quarterly basis on the development of possible successors to avoid delayed appointments when successors are likely to step into their roles. In addition to this, every manager is evaluated on one of the four People dimensions i.e. diversity and inclusion (D\&I). The company has focus on diversity lens while managing succession planning and it considers women and people from different geography with special attention so as to provide them equal developmental opportunities.

Moreover, HR has regular check on outcomes of performance systems to avoid imbalance based on sex, religion and racism. Additional aspects of leadership, motivation are also held answerable for contributing toward inclusive culture. All this analytics leads to championing diversified employee sub-group.

\section{CONCLUSION}

Employees being the most valuable resource of an organization so it becomes imperative that appropriate decisions are made in a vulnerable environment in which organization operates. With predictive capabilities of HR analytics, PepsiCo has been successful to establish itself as a robust organization taking competitive advantage. PepsiCo can be seen as a fascinating example of applying people analytics in routine decision-making and uncovering hidden patterns and insights about people at work. Analytics at PepsiCo has led some ingenious projects like strategic workforce planning, CEO pay ratio calculation, gender pay ratio calculation, long-term incentive for executive level employees and global headcount trends so as to make decisions in a shorter time frame. Conclusion is that Analytics being 1000 steps of journey but basically it starts from standardizing HR systems and process, integrating data across the borders and finally asking the right questions based on historical data that what PepsiCo has done so far. This is how PepsiCo has transformed and imbibed data drive approach in decision making.

\section{IMPLICATIONS FOR FUTURE}

From the above case of PepsiCo, People analytics can be considered a realistic practice and ongoing part of improved people impact on organizational performance. It is evident that People analytics should be looked up as an investment rather than an expense for the organization.
Indeed adopting people analytics is a costly affair for companies, but by outsourcing some of the People analytics services, it can reap the benefits of the same. The findings suggest that, People analytics has not only transformed internal operations but also increased productivity to support better decision making.

For enhanced performance and data driven decision making it is necessary to adopt people analytics as an integral part of organization. Future research may look for necessary quantitative data support, leadership qualities and competencies required to implement people analytics in context of Indian organizations. Further, the viability of application of People analytics in different sectors such as IT, Telecom, Manufacturing, Financial, Hospitality and Transportation can be anticipated. It would be more impactful if there existsextensive study on how analytics has changed People policies across the industries. Henceforth those findings of the study can be generalized.

\section{REFERENCES}

1. Bassi, L. (2011).Raging debates in HR analytics. People \& Strategy, 34(2), 14-18.

2. Buller, P. \& McEvoy, G. (2012).Strategy, human resource management \& performance: Sharpening line of sight.Human Resource Management Review. 22: 43-56.

3. Boudreau, J. W., \& Ramstad, P. M. (2006).Talent ship and HR Measurement and Analysis: From ROI to Strategic Organizational Change. Human Resource Planning, 29(1): 25-33. Retrieved February 23, 2012 from: http://stage.marshall.usc.edu/assets/048/9986.pdf

4. Cappelli, P. \&Tavis, A., (2018).HR Goes Agile. Retrieved from: https://hbr.org/2018/03/the-new-rules-of-talent-management

5. Carlson K. \& Kavanagh, M. (2015).In Kavanagh, M., Thite, M. \& Johnson, R. eds. Human resource information systems.Third edition. SAGE Publications: Thousand Oaks, California.

6. CiGen RPA (2019). 8 Real World Use Cases for Robotic Process Automation (RPA) in HR.

7. Retrieved from: https://medium.com/@cigen_rpa/8-real-world-usecases-for-robotic-process-automation-rpa-in-People-2f5724c88909

8. Deloitte (2013).Resetting Horizons Human Capital Trends

9. .Retrieved

from :https://www2.deloitte.com/content/dam/Deloitte/global/Documents/ HumanCapital/dttl-hc-hctrendsglobal-8092013.pdf

10. Deloitte (2016).Global Human Capital Trends, The new organization: $\begin{array}{lll}\text { Different by design.Retrieved } & \text { from }\end{array}$ :https://www2.deloitte.com/content/dam/Deloitte/global/Documents/ HumanCapital/gx-dup-global-human-capital-trends-2016.pdf

11. Goodwin, B., (2018). PepsiCo hires robots to interview job candidates. Retrieved from: https://www.computerweekly.com/news/252438788/PepsiCo-hiresrobots-to-interview-job-candidates

12. Hota, J., \& Ghosh, D. (2013).Workforce Analytics Approach: An Emerging Trend of Workforce Management. Workforce management, 167-179. Doi: 10.1002 /9781118636442.ch14

13. Lawler, E. E., Levenson, A., \& Boudreau, J. (2004).HR Metrics and Analytics Uses and Impacts.CEO Publication - Working Paper. Retrieved from :http://classic.marshall.usc.edu/ assets/048/9984.pdf

14. Mayo, A. (2006). Measuring and reporting: The fundamental requirement for data. CIPD - Research Report. Retrieved from http://www.mayolearning.com/mayo-publications/

15. Pfeffer, J., \& Sutton, R. I. (2006).Evidence-Based Management.Harvard Business Review, 84(1): 62-74. Retrieved from: http://jeffreypfeffer.com/wp-content/uploads/2011/10/HBRJan2006.pdf

16. PepsiCo automates invoice processing with ABBYY FlexiCapture. (n.d.). Retrieved from:https://www.abbyy.com/en-au/casestudies/pepsico-automates-invoice-processing-thanks-to-abbyyflexicapture/\#sthash.c163E1ej.dpbs

17. PepsiCo People Processes [PDF File] (2010). Retrieved from: https://www.catalyst.org/wp- 
content/uploads/2010/07/pepsico_People-processes_web_final.pdf
18. Search
Fortune
500
(2019). Retrieved
from: https://fortune.com/fortune500/2018/search/

19. Ulrich, D. \& Dulebohn, J.(2015). Are we there yet? What's next for HR?Human Resource Management Review. 25: 188-204.

20. Wainewright, P., (2017). Five takeaways from PepsiCo on moving SAP HR to the cloud.Retrieved from: https://diginomica.com/fivetakeaways-from-pepsico-on-moving-sap-People-to-the-cloud

\section{ABOUT THE AUTHORS}

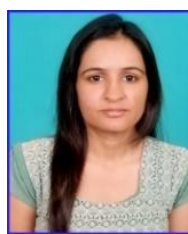

Rekha Bishnoi is a Research Scholar at the School of Management, The NorthCap University, Gurugram (Haryana). Currently she is pursuing research in the domain of human resources.

Her areas of interest are People Analytics, Workforce Analytics and Rewards and Incentives.

Rekha Bishnoi can be contacted at: rekhabishnoi3@gmail.com.

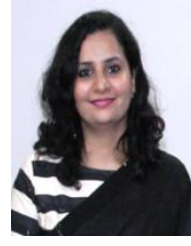

Dr. Poonam Arora is an Assistant Professor at The NorthCap University. She has 10 years' experience in academia and industry. Dr Poonam Arora's qualifications include $\mathrm{a} \mathrm{PhD}$ in the area of Organizational Commitment, MBA(HR). She has 8 years' teaching experience, including corporate experience. She has written more than 10 research papers and case studies for prestigious international journals. She can be contacted at: poonam2127@gmail.com 